\title{
Measurements of regional cerebral blood flow and cognitive performance in Alzheimer's disease
}

\author{
D Montaldi, D N Brooks, J H McColl, D Wyper, J Patterson, E Barron, J McCulloch
}

\begin{abstract}
Single photon emission computed tomography (SPECT) with $99 \mathrm{mTc}$ HMPAO was used to image 26 patients with dementia of the Alzheimer type (DAT) and 10 healthy controls. Regional cerebral blood flow (rCBF) data indicated a relative sparing of the occipital regions in DAT. Normalisation to occipital flow illustrated highly significant CBF deficits in a number of cortical regions, particularly in the left and right posterior-temporal cortex in DAT compared to controls. The cognitive performance of DAT patients was measured using a clinical cognitive assessment procedure (CAMCOG) and numerous correlations between these scores and rCBF were obtained. The implications and value of this investigative technique are discussed.
\end{abstract}

Alzheimer's disease is the most prominent form of dementia today affecting $5 \%$ of those over the age of 65 and $25^{\circ}$ of those over 80 years old. ${ }^{1}$ The cognitive deficits resulting from this disease can range from more focal deficits to an overall intellectual decline. ${ }^{23}$ The pathophysiological changes accompanying Alzheimer's disease involve both reductions in neurotransmitters and cell loss particularly in the medial temporal and frontal regions. ${ }^{4}$ In vivo imaging studies of the disease using positron emission tomography (PET) and single photon emission computed tomography (SPECT) techniques have analysed patterns of cerebral metabolism and regional cerebral blood flow ( $\mathrm{rCBF}$ ) respectively. The results of such studies reveal reductions in metabolism and flow in many cortical regions,,$^{5-7}$ however, parietal reductions are those most frequently reported. ${ }^{5-9}$

The aims of this study were three-fold. Firstly to attempt to replicate some previous findings using a dedicated tomographic brain scanner and radiopharmaceutical and a novel quantitation technique. Secondly, to identify those cerebral regions where bloodflow is particularly reduced in our Alzheimer population. Finally to relate these blood flow data to cognitive performance.

Table 1 Patient and control details

\begin{tabular}{|c|c|c|c|c|c|c|c|c|c|c|}
\hline & \multirow[b]{2}{*}{$N$} & \multicolumn{2}{|l|}{ Age } & \multicolumn{2}{|c|}{$M M S E$} & \multicolumn{2}{|c|}{ CAMCOG } & \multirow[b]{2}{*}{$\begin{array}{l}\text { Praxis } \\
\text { Mean }\end{array}$} & \multirow[b]{2}{*}{$\begin{array}{l}\text { Mem } \\
\text { Mean }\end{array}$} & \multirow[b]{2}{*}{$\begin{array}{l}\text { Lang } \\
\text { Mean }\end{array}$} \\
\hline & & Mean & Range & Mean & $(S D)$ & $\begin{array}{l}\text { Total } \\
\text { Mean }\end{array}$ & $(S D)$ & & & \\
\hline $\begin{array}{l}\text { DAT } \\
\text { Controls }\end{array}$ & $\begin{array}{l}26 \\
10\end{array}$ & $\begin{array}{l}76 \\
71\end{array}$ & $\begin{array}{l}54-90 \\
50-80\end{array}$ & $\begin{array}{r}9 \cdot 6 \\
28 \cdot 0\end{array}$ & $\begin{array}{l}(5 \cdot 5) \\
(1 \cdot 8)\end{array}$ & $\begin{array}{l}35.0 \\
94 \cdot 8\end{array}$ & $\begin{array}{r}(19 \cdot 7) \\
(6 \cdot 6)\end{array}$ & $\begin{array}{r}5 \cdot 1 \\
11 \cdot 0\end{array}$ & $\begin{array}{r}5.0 \\
23 \cdot 8\end{array}$ & $\begin{array}{l}15 \cdot 3 \\
27 \cdot 7\end{array}$ \\
\hline
\end{tabular}

\section{Methods and procedure}

All patients and controls form part of a longitudinal investigation into Alzheimer's disease involving six-monthly assessments on a series of cognitive tests and measurements of $\mathrm{rCBF}$ using SPECT.

Patient population. Twenty six patients (17 females) diagnosed as having dementia of the Alzheimer type (DAT) were studied (table 1). The mean age of the group was 76 years (5490). Diagnosis of Alzheimer's disease remains a serious problem; one which was tackled by applying a very strict classification system using the Cambridge Diagnostic Examination for the Elderly (CAMDEX) developed by Roth et al (1986).$^{10}$ Briefly, the CAMDEX criteria include a gradual intellectual deterioration for at least six months with the exclusion of reversible dementias, other psychiatric disorders (for example alcohol abuse, major depression), other neurological diseases (for example, multi-infarct dementia, communicating hydrocephalus), diabetes and malignancy and cases of severely impaired hearing or sight. Apart from standard laboratory tests, all patients also received ECG, EEG, and CT scans. Patients were selected for the DAT group only when no other possible cause of dementia could be considered. Although the patients varied in both severity and age at onset, they were grouped together for the purpose of this study.

Control population. Ten normal elderly controls (eight females) who were recruited from a local social centre were screened medically before entry into this group. The mean age of the group was 71 years (50-80). Informed consent was obtained for all patients and controls.

Neuroimaging technique. The radiopharmaceutical (Technetium-99m labelled HMPAO) was prepared by adding $1200 \mathrm{MBq}$ of $99 \mathrm{~m}$-Tc pertechnetate in $5 \mathrm{ml}$ saline to the freeze-dried mixture of the ligand (Ceretec, Amersham International). The patient dose of $500 \mathrm{MBq}$ was injected intravenously while the patient was sitting in a quiet room with eyes closed and ears unplugged.

HMPAO is readily removed from the blood during its first pass through the brain." Thereafter, a fast decomposition of the molecule takes place causing entrapment in the 
brain and producing a distribution which reflects $\mathrm{rCBF}^{12}$

Imaging was carried out five minutes to one hour after injection and was performed on a Novo 810 dedicated neuro SPECT imager. The patient lay unrestrained on the imager couch during the scanning session which lasted on average 20 to 25 minutes. During this time five slices, $12 \mathrm{~mm}$ thick, were obtained in a plane parallel to the orbital-meatal line and at positions $30,40,50,60$ and $70 \mathrm{~mm}$ superior to this line. Acquisition time for each slice was three minutes.

The Novo 810 imager uses the Harvard multi-detector scanning design utilising 12 sets of scintillation detectors with focussing collimators, arranged at $30^{\circ}$ intervals around the field of view. Each detector scans both tangentially and radially to the field of view resulting in relatively uniform spatial resolution, sensitivity and slice thickness throughout. the slice. The image quality of the brain tomographs produced are comparable with those produced by most PET scanners currently in use.

The five acquired images were compared with the anatomical patterns presented in a typical brain atlas for the purpose of defining two particular anatomical levels and for the identification of different brain regions. The lower of the two slices (the Standard slice), most usually at $\mathrm{OM}+40$, was defined by the presence of the putamen, thalamus and occipital cortex and by the absence of cerebellum. The high slice (usually $\mathrm{OM}+70$ ) lay immediately superior to the corpus callosum, and typically appeared as an elliptical ring of high activity in the cortex with a further line of high activity running continuously anterior to posterior representing cortex on each side of the inter-hemispheric fissure.

Regions of interest. Using the scanner's computer system, 14 regions of interest (ROI) outlining different brain regions were then drawn on each of these slices. (figs 1 and 2) Ten regions of interest (five on each side) were drawn on the standard slice and four on the high slice, with a separate outline encompassing the whole cerebral activity in both cases.

Corresponding right and left regions were symmetrically identical. The ROI boundaries were drawn along the outside surfaces of the brain and internally followed the division between the "grey" and "white" matter. On the standard slice the five regions were described as frontal, temporal, posterior temporal, occipital (fig 1), and basal ganglia; with high frontal and parietal on the high slice (fig 2 ). For each region the area and mean number of counts per pixel were measured and expressed as a proportion of the activity in the slice as a whole (Nmean). To normalise these data further we divided the Nmean for each ROI by the average Nmean of the occipital regions therefore producing an ROI/occipital activity ratio (Nmean po). The cerebrum/cerebellum ratio used in other studies $^{14}$ was not used here for two reasons. Firstly, using a dedicated neuro section scanner, a single image can be obtained within three minutes. This facility for rapid data collection

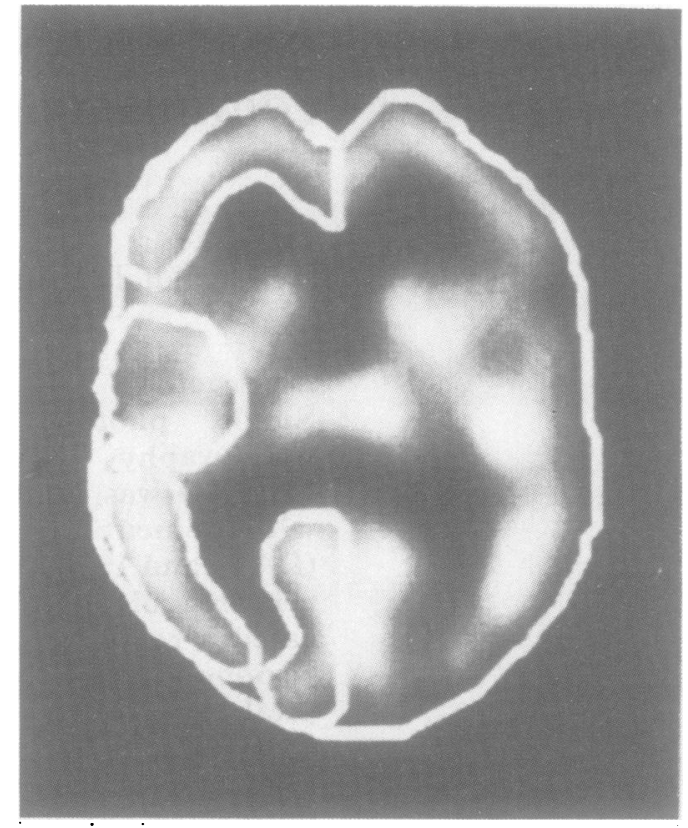

Figure 1 SPET image of Standard slice, illustrating regions of interest.

provides a distinct advantage over the gamma camera in studies of dementia since refusal rates can be high with this patient population. The images used to extract cortical data cannot include the cerebellum and therefore additional slice images would be required thus significantly increasing the imaging time and thereby prejudicing our very low refusal rate of $2 \%$. The second reason for not adopting the cerebellum for normalisation is that cerebellar blood flow may be affected by crossed cerebellar diaschisis resulting from damage to the cortico-ponto-cerebellar system. ${ }^{15}$ Selection of occipital flow as the best indicator of unimpaired flow was based on reports of a relative sparing of the occipital lobes in $\mathrm{DAT}^{8}$ a finding strongly supported by some preliminary results of our own.

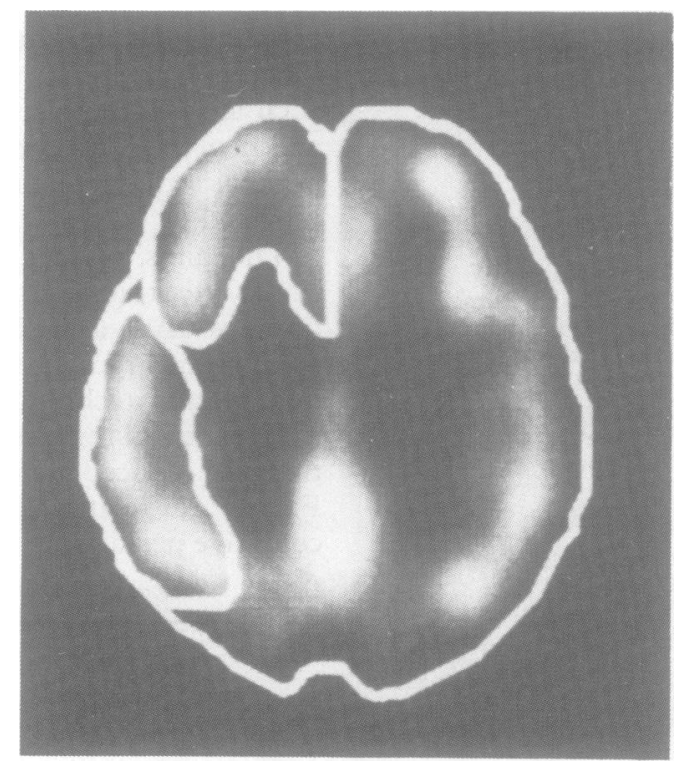

Figure 2 SPET image of High slice, illustrating regions of interest. 


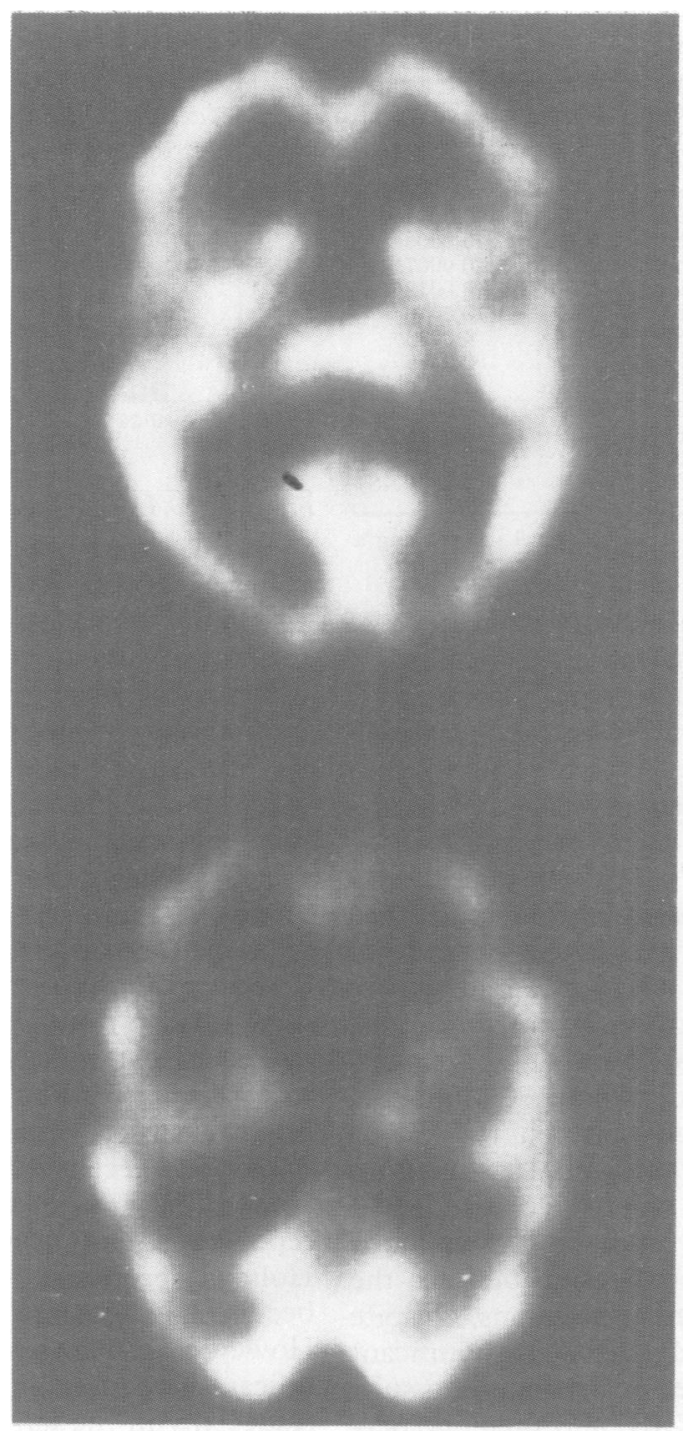

Figure 3 SPET scan illustrating blood flow in Standard slice of DAT (bottom) and control (top).

Examples of images. The images shown in figs 3 and 4 are SPECT scans of a patient with DAT, whose diagnosis was confirmed at necropsy, (bottom) and a normal control (top). Figure 3 illustrates blood flow in the Standard slice indicating reductions in frontal, temporal and posterior temporal flow together with a relative occipital sparing in patients with DAT. Figure 4 illustrates blood flow in a High slice which apart from showing a deficit in higher frontal flow, indicates a clear reduction in parietal blood flow in the DAT patient.

Cognitive assessment. Before carrying out a full neuropsychological battery, cognitive status was measured using the CAMCOG; a component of the CAMDEX. The CAMCOG is a short mental status examination similar to the Mini-Mental State Examination $(M M S E)^{13}$ which is included in its design. It is, however, more extensive than the MMSE in the areas of cognition that it covers. It comprises the following eight subcomponents:
1 Orientation
5 Memory
2 Language
3 Attention
4 Praxis
6 Calculation
7 Abstract thinking
8 Perception

The CAMCOG can either provide a single total score of overall cognitive performance, or

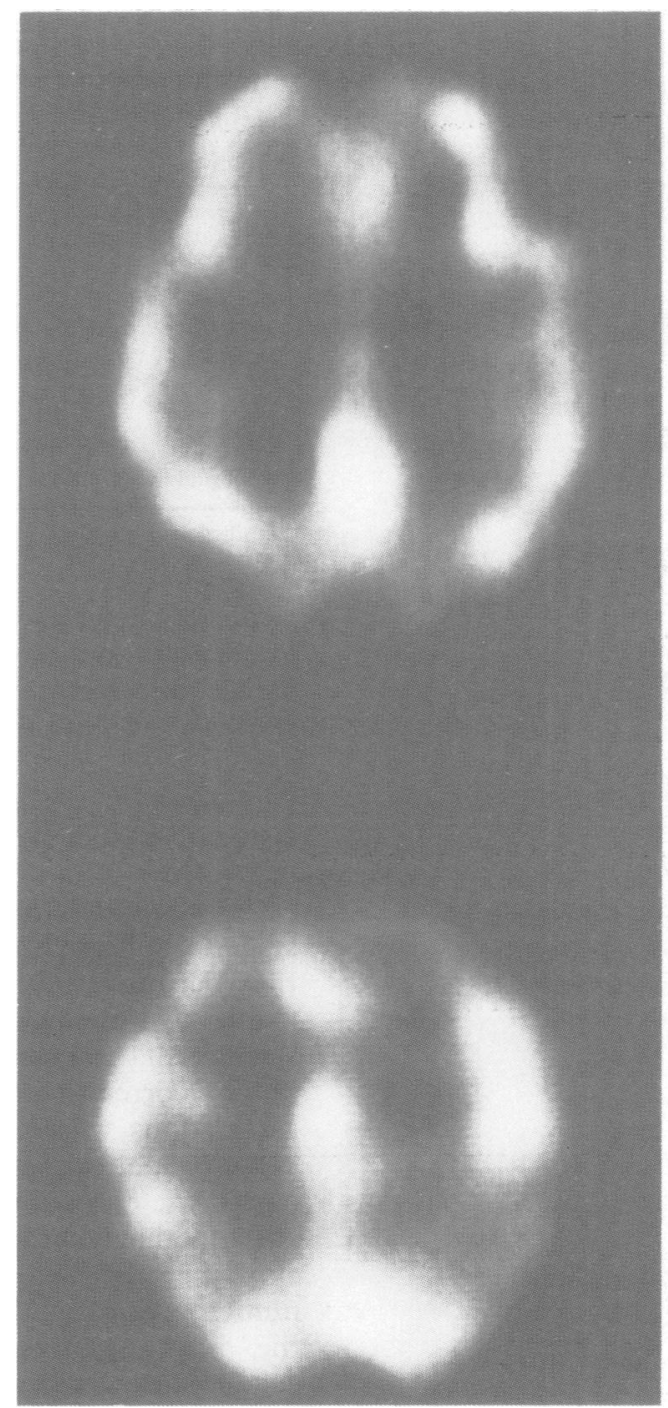

Figure 4 SPET scan illustrating blood flow in High slice of DAT (bottom) and control (top).

it can be broken down into subscores. For the purpose of this study we considered the total score and three subscores; Language, Memory and Praxis. We chose these subcomponents for two reasons: firstly, they are three cognitive functions known to be affected by DAT; and secondly, they are the three largest subcomponents of the CAMCOG. Administration of the CAMCOG -takes approximately half an hour and was carried out within two weeks of scanning.

\section{Results}

Patterns of $r C B F$

Before starting the formal analysis we compared $\mathrm{rCBF}$ in DAT and controls with flow normalised to the slice alone. This showed a highly significant sparing of the occipital regions in DAT, lending support to our decision to study flow normalised to the occipital cortex.

The first part of our data analysis was designed to compare rCBF in the DAT group to that of controls. To do this we used two sample $t$-tests to compare the ROI/occipital ratios for each ROI across the two groups, and the results are described in table 2 . As the pvalues clearly show there is strong evidence of 
Table 2 Regional cerebral blood flow levels in patients with $D A T$ and normal controls

\begin{tabular}{llll}
\hline & \multicolumn{2}{l}{ Nmean po } & \\
\cline { 2 - 3 } ROI & $D A T$ & $N C$ & p-value \\
\hline L posterior-temporal & 0.9 & 1.07 & $<0.0001^{\star}$ \\
R posterior-temporal & 0.89 & 1.01 & $0.0006^{\star}$ \\
L temporal & 0.84 & 0.98 & $0.0007^{\star}$ \\
L parietal & 0.93 & 1.04 & $0.0012^{\star}$ \\
R frontal & 0.83 & 0.96 & $0.0016^{\star}$ \\
L frontal & 0.82 & 0.94 & $0.0023^{\star}$ \\
R parietal & 0.92 & 1.00 & 0.0055 \\
R temporal & 0.87 & 0.98 & 0.0090 \\
R high frontal & 0.84 & 0.96 & 0.0090 \\
L high frontal & 0.85 & 0.96 & 0.0115 \\
L basal ganglia & 1.02 & 1.12 & $0.060(\mathrm{NS})$ \\
R basal ganglia & 1.02 & 1.08 & $0.125(\mathrm{NS})$ \\
\hline
\end{tabular}

$\star$ Significance obtained at an overall $5 \%$ level when using a Bonferroni correction.

reduced blood flow in all cortical regions of interest, but not the basal ganglia. The top $6 \mathrm{p}$ values, however, produced particularly strong evidence since significance was still obtained at an overall $5 \%$ level when applying a Bonferroni correction (a very conservative statistic greatly reducing chance significance).

$r C B F$ and cognitive performance. The final stage of our analysis investigated the association between rCBF data and cognitive performance in the DAT group. We measured the strength of these associations using Spearman's rank correlation coefficient. Numerous associations were found between rCBF and cognitive performance, and table 3 includes not only those correlations where significance was still obtained at an overall 5\% level when applying a Bonferroni correction but also the numerous less significant associations. Despite the large number of strongly significant correlations, there were, surprisingly, no such correlations between rCBF and memory performance. This lack of strong correlations with memory was investigated further by analysing CAMCOG total, and subscore distributions. As fig 5, 6 and 7 illustrate, the total score, and language and praxis scores all show a broad distribution. The memory scores, however, show no such distribution, having a marked floor-effect (fig 8). Since correlational analysis requires a reasonable distribution of scores to produce meaningful results, the absence of correlations with memory reflects this flooreffect.

\section{Discussion}

Regional cerebral bloodflow measurement

Table 3 Positive associations between cognitive performance and $r C B F$

\begin{tabular}{lllll}
\hline ROI & CAMCOG & Memory & Language & Praxis \\
\hline Rf & $\star$ & & $\star$ & $\star$ \\
Lf & $\star$ & & $\star$ & $\star$ \\
Rt & $\star$ & & $\star \star$ & $\star$ \\
Lt & $\star \star$ & $\star$ & $\star \star$ & $\star$ \\
Rpt & $\star \star$ & $\star$ & $\star \star$ & $\star \star$ \\
Lpt & $\star \star$ & & & $\star$ \\
Rhf & & $\star$ & $\star$ & $\star$ \\
Lhf & $\star \star$ & $\star$ & $\star \star$ & $\star \star$ \\
Rp & $\star \star$ & $\star$ & & \\
Lp & & & & \\
\hline
\end{tabular}

$\star$ Correlations significant at an individual $5 \%$ level. $\star \star$ Correlations significant at an overall $5 \%$ level when applying a Bonferroni correction.

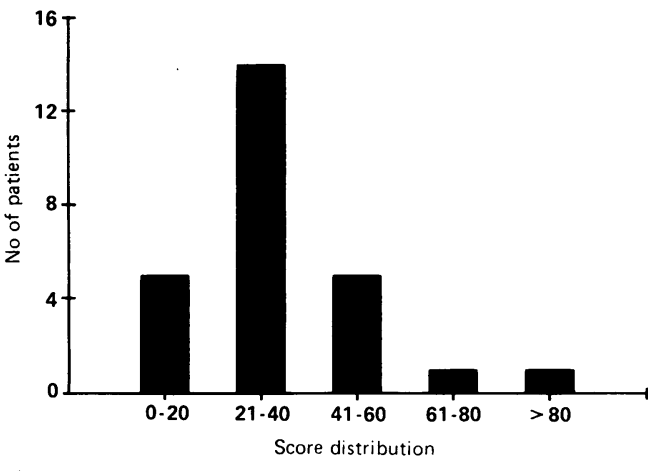

Figure 5 CAMCOG: Total scores.

using the NOVO 810 tomograph and $99-\mathrm{m} \mathrm{Tc}$ HMPAO produces significantly different results in patients with Alzheimer's disease when compared to elderly controls. Using a normalised mean value of bloodflow we replicated the finding of a relative sparing of the occipital lobes ${ }^{8}$ and proceeded to use this to further normalise our data. Using this technique we have provided strong evidence of the involvement of the posterior temporal regions in Alzheimer's disease. While there appear to be no specific reports of $\mathrm{CBF}$ deficits in this region, these results provide extremely strong evidence that it is clearly implicated in the disease process. The well documented reduction in parietal blood flow in Alzheimer's disease has received further support from this study. The parietal regions are so frequently reported as implicated in the disease that Holman proposed that their damage has become the hallmark of Alzheimer's disease. ${ }^{16}$ However, while parietal bloodflow is significantly reduced in our patient population, (especially in the left hemisphere), the results of this study suggest that there is an even greater involvement of both the left and right posterior temporal regions in this disease.

The involvement of the frontal lobes in Alzheimer's disease provokes considerable debate. There is no doubt that in many cases of DAT, a degree of frontal pathology exists. ${ }^{7} 1718$ However, many researchers like to separate patients with frontal damage and label them as "frontal dementias" or possible Pick's disease. ${ }^{19}$ Our results identify a frontal component in DAT since the rCBF deficits were highly significant in both the left and right frontal regions of a population showing no disproportionate frontal signs clinically. Foster, Chase and $\mathrm{Mansi}^{18}$ suggest that anterior changes

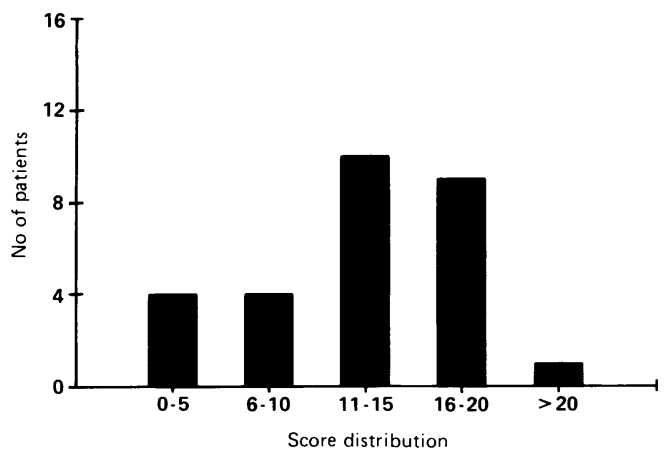

Figure 6 CAMCOG: Language scores. 
Figure 7 CAMCOG: Praxis scores.

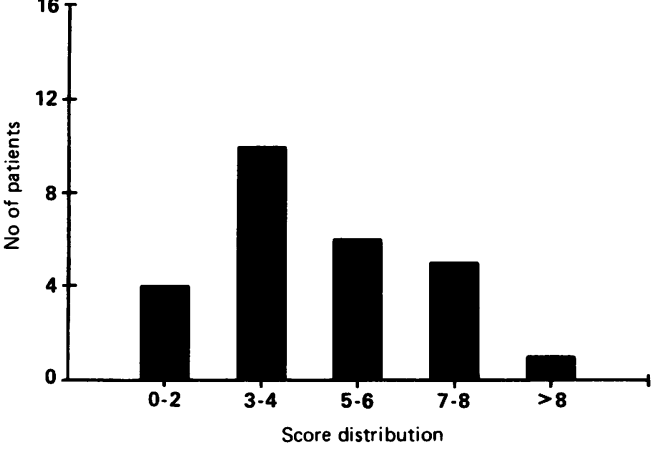

occur late on in the disease process, and this suggestion is supported by our findings, since the majority of our patients are moderately to severely demented, (See MMSE scores table 1). This does not, of course, exclude the possibility that minimal/mild cases of DAT could show some frontal rCBF deficits. We would therefore suggest that the developing trend towards using SPECT evidence of extensive frontal pathology as an exclusion criterion for Alzheimer's disease, ${ }^{20}$ is unwise and may mask interesting features of this disease. There may exist for example, a "frontal" subgroup in the same way as Martin et al identified a "parietal" and a "temporal" subgroup on the basis of both cognitive and metabolic profiles.

Turning now to discuss the relationship between SPECT data and cognitive performance in DAT. While the highly significant correlations (ranging from $0: 57$ to $0 \cdot 74$ ), between $\mathrm{rCBF}$ and cognitive performance as measured by the CAMCOG, support current theories of cortical organisation and cognitive function, there are two problems which make interpretation of the results particularly difficult. The first problem is that of multiple correlations. Apart from the thirteen very highly significant correlations, numerous less significant associations were also found. This multiplicity of correlations prevents the drawing of firm conclusions about the specific relationship between rCBF deficits and cognition in this population. The second problem is that in spite of the strength of some of the correlations and the extensively reported memory deficit accompanying DAT, we obtained no such correlations between rCBF and memory performance on the CAMCOG.

To avoid such confounding results in future research we offer two alternative explanations for these problems. The first of these is that the CAMCOG and other cognitive screening tests are inappropriate tools for this form of investigation. Due to the requirements of "brief"

Figure 8 CAMCOG: Memory scores. cognitive screening tests, such procedures are, by nature non-specific and the subtests may frequently overlap in the cognitive domains they are assessing. Therefore, while this type of assessment tool is ideal for providing a general cognitive picture, much greater task specificity is required if meaningful correlations are to be sought. The inappropriate use of the CAMCOG could therefore be responsible for the excessive number of correlations found in this and other studies. ${ }^{1421}$ Interestingly, this lack of task specificity may also account for the failure to find correlations between $\mathrm{rCBF}$ and memory. The non-specific nature of screening tests greatly reduces their sensitivity and capacity to identify subtle differences in cognitive performance between patients. This situation is clearly illustrated here by the flooreffect we found with the memory component of the CAMCOG (fig 8). Despite evidence for differential memory deficits in dementia (for example, visual/verbal, implicit/explicit); memory on this test can only be expressed as one single score since subcomponent scores would be too small. Again, while these subcomponents adequately assess memory for screening procedures they are far too generalised (for example, they measure almost exclusively verbal memory), for use in correlational studies of this form. Without the use of specific tasks, floor-effects or similar confounding features will prevent both an accurate analysis and a useful interpretation of results. Thus, use of this type of tool to provide evidence of subgroups, ${ }^{14}$ particularly for studies of the heterogeneity of Alzheimer's disease, is very questionable and highly inadvisable.

The second explanation for our results could be the nature of our patients who are typically in the later stages of the disease. As with the reductions in blood flow, the cognitive impairments resulting from the later stages of the disease are diffuse and appear to affect nearly every cognitive domain and numerous significant correlations may therefore be expected. Unfortunately reports of similar studies also producing multiple correlations ${ }^{14}{ }^{21}$ do not describe the severity of the population under investigation. So far as the floor effect on memory is concerned, it could be argued that this may have resulted solely from the severe nature of our patient population. However, some preliminary results from our neuropsychological battery suggest that the lack of task specificity provides a much more plausible explanation. Although the memory component of the CAMCOG produced a floor-effect, the same moderate to severe patient population reported here produced a substantial distribution of scores on more specific tasks such as visual memory $\operatorname{span}^{22}$ and paired associate learning. ${ }^{23}$ This enables us to investigate more specific relationships between $\mathrm{rCBF}$ and memory, while avoiding the problem of multiple correlations which swamp any specific effects.

We have illustrated rCBF deficits in a moderate to severely demented population suffering from DAT, using HMPAO and SPECT. In particular, we have identified the 
posterior temporal regions as those showing the largest blood flow deficits, while a relative sparing is indicated in the occipital regions. Certainly the clinical and cognitive severity of the patient population must always be considered when designing a study, and subsequently reported in publications. Most importantly, however, non-specific tools such as the CAMCOG can produce results which are difficult to interpret and which may lead the unwary to oversimplistic and inaccurate conclusions. In the case of this study and others like it $^{1421}$ we have used very sophisticated means of measuring rCBF, yet extremely crude means of measuring cognitive performance. In future this type of research should adopt cognitive assessment tools compatible in sophistication to that of the neuroimaging technique.

This study was funded by The Wellcome Trust Grant 18738/1.19.

We thank Mary-Teresa Hansen and Shona Wylie for their technical and nursing assistance.

1 Terry RD, Katzman R. Senile Dementia of the Alzheimer Type. Annals of Neurology 1983;14:497-506.

2 Huppert FA, Tym E. Clinical and neuropsychological assessment of Dementia. Br Med Bull 1986;42 No 1 11-18.

3 Martin A, Browers P, Lalonde F, et al. Towards a Behavioural Typology of Alzheimer patients. J Clin Exp Neuropsychol 1986;8 No 5:594-610.

4 Van Hoesen GW, Damasio A. Neural correlates of cognitive impairment in Alzheimer's Disease. In: Mountcastle VB Plum F, Geiger SR, eds. Handbook of Physiology 1987; Section 1, Vol 5:871-98.

5 Bonte FJ, Elliott DR, Hazem CH, Devous Sr. MD. Spect Study of Regional Cerebral Bloodflow in Alzheimer's Disease. Journal of Computer Assisted Tomography 1986;10(4):579-83.

6 Johnson KH, Mueller ST, Walshe TM, et al. Cerebral perfusion imaging in Alzheimer's Disease with SPECT and I-123 IMP. Neurology 1985;35 (Suppl 1):235.

7 Duara R, Grady C, Haxby JV, et al. Positron emission tomography in Alzheimer's Disease. Neurology

8 Risberg J. Cerebral blood flow in dementias. Danish Med Bull 1985;32(Suppl 1):48-50.

9 Frackowiak RSJ, Pozzili C, Legg NJ, et al. Regional cerebra oxygen supply and utilization in dementa: A clinical and physiological study with oxygen-15 and positron tomography. Brain 1981:104:753-78

10 Roth M, Tym E, Mountioy CQ, et al. CAMDEX: A standardised instrument for the diagnosis of mental disorder in the elderly with special reference to the disdetection of dementia $B r$ special reference to the early

11 Andersen AR, Friberg H, Knudsen K, et al. Extraction of ${ }_{99 m}$ Tc d-L-HMPAO across the blood brain barrier. $J$ Cerebral Blood Flow Metab 1988:8:544-51.

12 Neirinckx RD, Canning LR, Piper IM, et al. Technetium 99m d-1 HMPAO: A new radiopharmaceutical for SPECT Imaging regional cerebral blood perfusion. $J \mathrm{Nucl}$ Med 1987;28:191-202.

13 Folstein MF, Folstein SE, McHugh PR. "Mini-mental state" a practical method for grading the cognitive state of patients for the clinician. J Psychiatr Res 1975;12:189-98.

14 Burns A, Philpot MP, Costa DC, et al. The Investigation of Alzheimer's disease with single photon emission tomography. J Neurol, Neurosurg Psychiatry 1989;52:248-53.

15 Baron JC, Bousser, MG, Comar D, Castaigne P. Crossed cerebellar diaschisis in human supratentorial infarction. Ann Neurol 1980a:8:128.

16 Holman BL. Perfusion and receptor SPECT in the dementias. George Taplin Memorial Lecture. J Nucl Med 1986;27:855-60.

17 Grady CL, Haxby B, Horwitz M, et al. Longitudinal study of the early neuropsychological and cerebral metabolic changes in dementia of the Alzheimer type. J Clin Exp Neuropsychol 1988;10,(5):576-96.

18 Foster NL, Chase TN, Mansi L, et al. Cortical abnormalities in Alzheimer's Disease. Ann Neurol 1984;16:649-54.

19 Neary D, Snowden JS, Shields RA, et al. Single photon emission tomography using ${ }^{99 m} \mathrm{TC}-\mathrm{HM}-\mathrm{PAO}$ in the investigation of dementia. J Neurol, Neurosurg Psychiatry 1987;50:1101-9.

20 Neary D, Snowden JS, Goulding P. Dementia of frontal lobe type. In: J Neurol Neurosurg Psychiatry 1988;51:353-61.

21 Hunter R, McLuskie R, Wyper D, et al. The pattern of function-related regional cerebral blood flow investigated by single photon emission tomography with ${ }^{99 m} \mathrm{Tc}$ HMPAO in patients with presenile Alzheimer's disease and Korsakoff's psychosis. Psychol Med (In press).

22 Wilson L, Brodie E, Reinink E, et al. Memory for Pattern and Path in Senile Dementia. J Clin Exp Neuropsychol 1988;10(1):77.

23 Isaacs B, Walkey FA. A simplified Paired-Association Test for Elderly Hospital Patients. Br J Psychiatry 1964:110:80-3 